\title{
CRIOGLOBULINEMIA ESENCIAL COMO CAUSA DE HEMORRAGIA ALVEOLAR - REPORTE DE CASO Y REVISIÓN DE LA LITERATURA
}

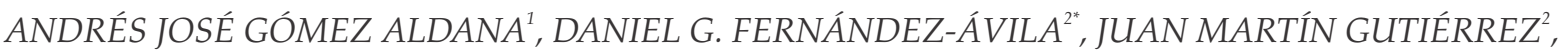 \\ MARÍA CLAUDIA DÍAZ \\ ${ }^{1}$ Médico Internista, Pontificia Universidad Javeriana - Hospital Universitario San Ignacio \\ ${ }^{2}$ Médico Internista Reumatólogo, Pontificia Universidad Javeriana - Hospital Universitario San Ignacio. Grupo \\ Javeriano de Investigación en Enfermedades Reumáticas \\ *Correspondencia: Daniel G. Fernández Ávila. daniel.fernandez@javeriana.edu.co \\ Dirección Postal: Carrera 7 No. 40 - 62. Hospital Universitario San Ignacio
}

Recibido: 23 Mayo 2014 Aceptado: 10 Abril 2015

\begin{abstract}
Resumen
Presentamos el caso de una paciente quien ingresa por la presencia de lesiones petequiales, artralgias y una úlcera en miembro inferior. La presencia de azoados en ascenso progresivo se documenta durante su estancia. Se instaura manejo con corticoide IV sin respuesta y se obtienen mejoría con plasmaféresis. Posteriormente se observa la presencia de deterioro del patrón respiratorio, disnea sumado a la presencia de compromiso pulmonar en parches por medio de tomografía de tórax y con evidencia de hemorragia alveolar. Se descartan causas autoinmunes e infecciosas, así como neoplasias hematológicas. Se concluye que la presencia de crioglobulinemia es la única causa de dicha entidad.
\end{abstract}

Palabras clave: Crioglobulinas, hemorragia alveolar, insuficiencia renal, disnea, autoinmunidad.

\section{ESSENTIAL CRYOGLOBULINEMIA AS A CAUSE OF ALVEOLAR HEMORRHAGE - CASE REPORT AND LITERATURE REVIEW}

\begin{abstract}
We report the case of a patient who is admitted by the presence of petechial lesions, arthralgia and an ulcer in lower limb. The presence of progressively increasing creatinine is documented while staying. Unanswered management with corticosteroid IV was instituted and there was an improvement with plasmapheresis. Later, the presence of impaired breathing pattern was notice, dyspnea combined with the presence of lung involvement in patches through chest tomography and evidence of alveolar hemorrhage. Autoimmune and infectious causes and hematological malignancies were dismissed. The presence of cryoglobulinemia was identified as the unique cause of such entity.
\end{abstract}

Keywords: Cryoglubulins, alveolar hemorrhage, renal failure, dyspnea, autoimmunity. 


\title{
CRIOGLOBULINEMIA ESSENCIAL COMO CAUSA DE HEMORRAGIA ALVEOLAR
}

\begin{abstract}
Resumo
Presentamos o caso duma paciente que ingressa pela presencia de lesiones petequiales, artraigias $e$ uma úlcera no membro inferior. A presencia de azoados em ascensão progressivo documenta-se durante sua estancia. Instaura-se manejo com corticoide IV sim resposta e melhoram com plasmaféresis. Posteriormente, observou-se além a presencia de deterioro do padrão respiratório disnea a presencia de compromisso pulmonar em parches com tomografia de tórax e com evidencia de hemorragia alveolar. Descartem-se causas autoimunes e infecciosas, assim como neoplasias hematológicas. Conclui-se que a presencia de crioglobulinemia é a única causa de essa entidade.
\end{abstract}

Palavras-chave: Crioglobulinas, hemorragia alveolar, insuficiência renal, disnea, autoimunidade.

\section{Introducción}

La crioglobulinemia es una entidad de baja frecuencia de presentación, que se relaciona en muchos casos a otras entidades nosológicas, con un amplio espectro clínico de presentación. Presentamos el caso de una paciente quien presenta crioglobulinemia asociado a hemorragia alveolar.

\section{Reporte de caso}

Mujer de 57 años procedente de Bogotá quien consulta por cuadro clínico de 2 meses de evolución de aparición de úlcera dolorosa en pierna derecha (Figura 1.), acompañado de mialgias y artralgias de predominio en rodilla y articulaciones interfalángicas que disminuían con la actividad física, por lo que consultó inicialmente a médico general, quien inició manejo con corticoide tópico sin mejoría, presentando posteriormente secreción purulenta y fétida abundante, al igual que la presencia de múltiples lesiones purpúricas en piel de piernas y muslos (figura 2), solicitando paraclínicos iniciales los cuales documentan anemia normocítica normocrómica con $\mathrm{Hb}$ de $6.2 \mathrm{~g} / \mathrm{dl}$ VCM de $85.4 \mathrm{fl} \mathrm{y} \mathrm{CHCM} \mathrm{de} 34.5 \mathrm{~g} / \mathrm{dl}$ la cual fue manejada con transfusión de 2 unidades de glóbulos rojos empaquetados y control de $\mathrm{Hb}$ en $11.2 \mathrm{~g} / \mathrm{dl}$, con leucocitos de $11200 \mathrm{cel} / \mathrm{ul}$ y plaquetas de $139000 \mathrm{cel} / \mathrm{ul}$ considerando en dicha oportunidad la sospecha de sobreinfección bacteriana por lo que se instauró cubrimiento antibiótico con ampicilina sulbactam por 7 días.
Ante la presencia de dicha úlcera en miembro inferior derecho, se decide descartar etiología vascular de la misma, para lo cual se solicitó doppler arterial y venoso, los cuales fueron normales, con posterior realización de biopsia, la cual solo mostro úlcera vasculítica.

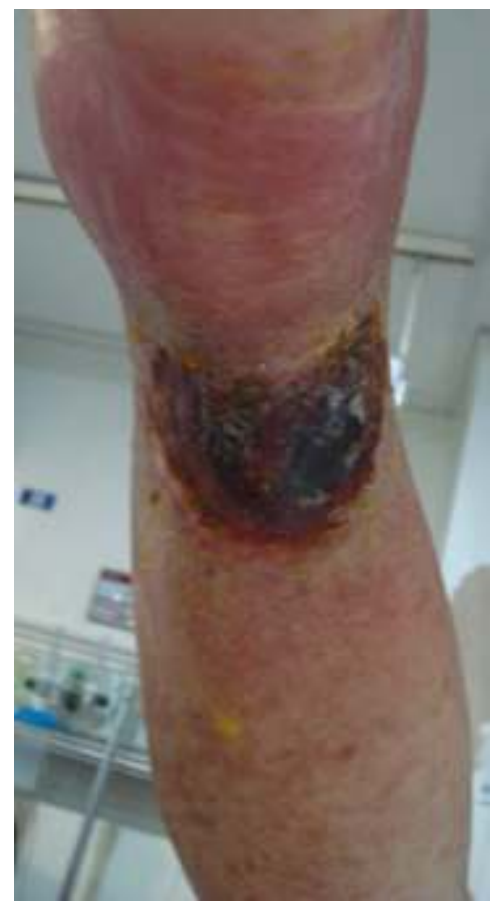

Figura 1. Úlcera en Miembro inferior derecho. Foto tomada por los autores. 


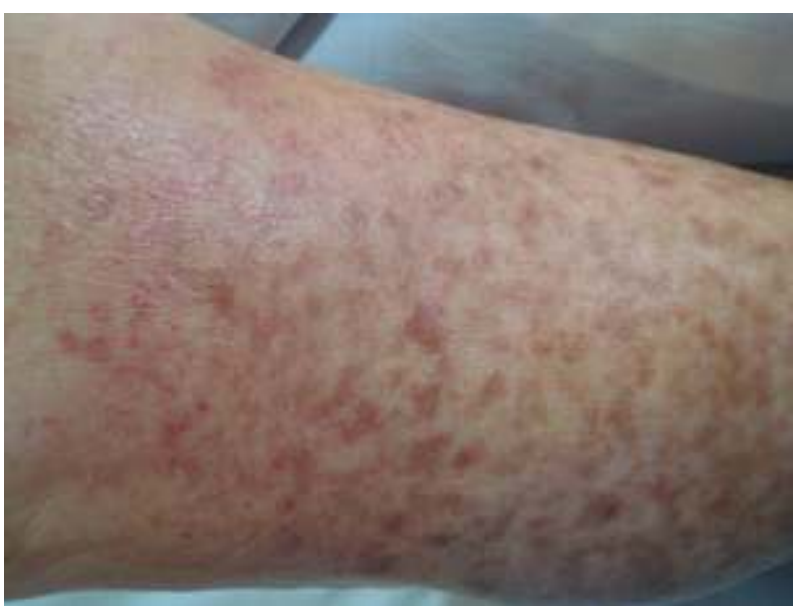

Figura 2. Lesiones purpúricas en pierna derecha. Foto tomada por los autores.

Adicionalmente, considerando la presencia de dolor articular, se solicitó factor reumatoide, obteniendo un resultado de $71.9 \mathrm{UI} / \mathrm{ml}$ (normal hasta $20 \mathrm{UI} / \mathrm{ml}$ ), con posterior toma de anticuerpos anticitrulínicos con un valor de $0.2 \mathrm{U}$, considerándolos negativos.

Sin embargo durante su estancia en la institución presenta oliguria persistente por más de 48 h (GU $0.35 \mathrm{cc} / \mathrm{kg} / \mathrm{h}$ ) acompañado de elevación de azoados con una creatitina de ingreso al hospital de $1.86 \mathrm{mg} / \mathrm{dl}$ y BUN de $34.6 \mathrm{mg} / \mathrm{dl}$, alcanzando a los dos días creatinina de $2.31 \mathrm{mg} / \mathrm{dl}$ y BUN de $54.3 \mathrm{mg} / \mathrm{dl}$ hasta alcanzar un valor de creatinina de 2.54 $\mathrm{mg} / \mathrm{dl}$ y BUN de $80 \mathrm{mg} / \mathrm{dl}$, con uroanálisis en el cual se evidencia proteinuria de $200 \mathrm{mg} / \mathrm{dl}$ en muestra aislada y hematuria con $10-20 \mathrm{cel} / \mathrm{campo}$, con realización de ecografía renal y de vías urinaria normal, depuración de creatinina en $24 \mathrm{~h}$ de $12.3 \mathrm{ml} / \mathrm{min}$, proteinuria en $24 \mathrm{~h}$ de $0.74 \mathrm{~g}$, por lo cual es valorada por el servicio de nefrología quienes consideran el diagnóstico de glomerulonefritis rápidamente progresiva por lo que instauran manejo con 3 pulsos de metilprednisolona $500 \mathrm{mg}$ a pesar de lo cual, continúa con ascenso de azoados, decidiéndose entonces manejo con plasmaféresis, con buena respuesta clínica dado por mejoría en la función renal, al igual que en el gasto urinario.

Simultáneamente con el deterioro de la función renal, la paciente presenta disnea progresiva y deterioro de la clase funcional con dificultad respiratoria súbita requiriendo intubación orotraqueal y traslado a la unidad de cuidados intensivos donde se toman paraclínicos, documentando la presencia de anemización con $\mathrm{Hb}$ de $6.6 \mathrm{~g} / \mathrm{dl}$ y Hto de 19.6 $\%$ por lo que se realiza radiografía de tórax en la cual se encuentran múltiples opacidades alveolares de predominio en bases pulmonares y tomografía de tórax de alta resolución que documenta extensa opacidad alveolar en la región central de ambos pulmones con vidrio esmerilado en los dos tercios superiores.

Ante tales hallazgos, se considera la necesidad de realizar fibrobroncoscopia la cual evidencia hallazgos compatibles con hemorragia alveolar, observando la presencia de hemosiderófagos en un $60 \%$ en el lavado broncoalveolar, por lo que se ratifica dicho diagnóstico.

Igualmente, se observa nueva elevación de azoados con creatinina de $2.47 \mathrm{mg} / \mathrm{dl}$ y BUN de $83.4 \mathrm{mg} / \mathrm{dl}$ con gasto urinario limítrofe de $0.47 \mathrm{cc} / \mathrm{kg} / \mathrm{h}$, por lo que considerando el compromiso simultáneo pulmonar y renal, sin documentar en el momento proceso infeccioso por ausencia de crecimiento de gérmenes en hemocultivos y aspirado de secreción traqueal, se decide el inicio de plasmaféresis alcanzando 5 sesiones observando mejoría paulatina de azoados con creatinina de $1.35 \mathrm{mg} / \mathrm{dl}$ y disminución de las opacidades alveolares documentadas en la radiografía de tórax. Se realizó posteriormente biopsia renal percutánea sin embargo no se logró obtener el reporte histológico de dicho procedimiento dado que se tomó una muestra insuficiente para hacer un diagnóstico histopatológico.

Dentro de los estudios diagnósticos que explicaran el compromiso renal y pulmonar con el cual estaba cursando la paciente, se decide descartar etiología autoinmune solicitando ANAS, ENAS, anti DNA los cuales fueron negativos, complemento normal, pANCAS y cANCAS negativos, anticardiolipinas IgG e IgM y anticoagulante lúpico negativos. El paciente fue valorado por reumatologia, considerándose que no cursaba con enfermedad del tejido conectivo. Adicionalmente, se decidió descartar la presencia de síndrome de Goodpasture, para lo cual se solicitaron anticuerpos anti membrana basal glomerular, los cuales fueron negativos. Finalmente, teniendo en cuenta el cuadro clínico de la paciente se consideró como opción diagnóstica la presencia de crioglobulinemia para lo cual se solicitó crioglobulinas las cuales fueron positivas (950 $\mathrm{mcg} / \mathrm{dl})$.

Ante este hallazgo que sugiere crioglobulinemia se decide definir la etiología de dichos hallazgos por lo se buscaron causas infecciosas con antigeno de superficie para hepatitis $B$ en $0.23 \mathrm{UI} / \mathrm{ml}$ siendo éste no reactivo, anticuerpos contra hepatitis $C$ en $0.09 \mathrm{~S} /$ co no reactivo, así como anticuerpos contra $\mathrm{VIH}$ con valor de $0.19 \mathrm{~S} / \mathrm{co}$ siendo no reactivo.

Igualmente, se consideró causa hematológica con realización de aspirado de médula ósea el cual solo evidencia disminución de la línea eritroide y línea granulocítica ligeramente aumentada, y citometría de flujo que documenta la presencia de linfocitos $\mathrm{B}$ policlonales del $2.5 \%$ con electroforesis de proteínas con un valor de Ig A de 
$124.5 \mathrm{mg} / \mathrm{dl}$ (valor normal 70-400), Ig G de $595 \mathrm{mg} / \mathrm{dl}$ (valor normal de 700 - 1600) e Ig M de 259 (valor de 40 230).

Se observa posteriormente evolución clínica estable con creatinina estable con un valor de $1.38 \mathrm{mg} / \mathrm{dl}$, con ausencia de sintomatología respiratoria y control del dolor articular, por lo que se decide continuar manejo ambulatorio con prednisolona oral con lo que la paciente se mantiene en remisión durante el seguimiento ambulatorio.

\section{Discusión}

Las crioglobulinas son las inmunoglobulinas que se precipitan in vitro en temperaturas bajas (menores de 37 ) $(1,2)$ siendo descrita su naturaleza patológica en 1933 por Wintrobe y Buell 3, documentando en un paciente con mieloma múltiple y con posterior descripción de la enfermedad crioglobulinémica por Meltzer y colegas en 1966 (1).

Estas moléculas generadas por el sistema inmune han sido clasificadas por Brout en 1974, usando dicha distribución desde entonces, basado en el análisis inmunohistoquímico, siendo las de tipo I, consistente en inmunoglobulinas monoclonales Ig M o Ig G con un 10 - 15\% de los casos, la tipo II es una mezcla de Ig $\mathrm{M}$ monoclonal e Ig $\mathrm{G}$ policlonal con un $50-60 \%$ del total, acompañándose de actividad del factor reumatoide, y la tipo III siendo una mezcla de Ig M e Ig G policlonal, considerando estas dos últimas categorías como parte de las crioglobulinemias mixtas $(2,3)$.

Las criobloguinemias mixtas se han asociado con múltiples entidades como enfermedades hematológicas malignas entre las que se han descrito la macroglobulinemia de Waldenstrom, mieloma múltiple y leucemia linfocítica crónica. , o procesos infecciosos como hepatitis B o C documentando en un estudio de pacientes italianos la presencia de un RNA de virus de hepatitis C en un 90\% de pacientes con crioglobulinemia mixta (4). Otras causas infecciosas descritas son la infección por Epstein Barr, virus de la inmunodeficiencia humana, citomegalovirus, leishmania o treponema entre otros, al igual que con enfermedades de naturaleza autoinmune como el síndrome de Sjogren, lupus eritematoso sistémico. Adicionalmente, cuando se documenta su presentación en causa de una enfermedad definida se considera como crioglobulinemia mixta esencial descrito en un $10 \%$ de los pacientes $(1,2,3)$.

Dentro de las características fisiopatológicas de esta enfermedad, llama la atención la expansión clonal de células $B$ que surge tanto en enfermedades linfoproliferativas, como en enfermedades autoinmunes o infecciones crónicas, como en la infección por hepatitis $\mathrm{C}$ en la cual se encuentra un linfotropismo por el virus generando la expansión clonal de linfocitos $\mathrm{B}$, por medio de la interacción de la proteína E2 de la envoltura viral con la molécula extracelular CD81, lo cual facilita la producción de inmunoglobulinas como Ig M (1).

Sin embargo un factor fundamental de la crioglobulinemia es la precipitación de las inmunoglobulinas a temperaturas bajas y fenómenos de oclusión vascular, que actúa como contribuyente de las manifestaciones clínicas de dicha enfermedad en extremidades distales, al igual que la precipitación de los complejos inmunes en los vasos.

Dentro de las manifestaciones clínicas de esta enfermedad se encuentra la presencia de la tríada caracterizada por púrpura, artralgias y debilidad, así como un espectro de signos de síntomas agrupados en diferentes entidades como el síndrome de hiperviscosidad documentado generalmente en crioglobulinemia tipo I el cual presenta compromiso neurológico dado por cefalea, visión borrosa con documentación al examen físico de hemorragias retinales, epistaxis, con falla renal, al igual que la evidencia de lesiones en piel como púrpura, fenómeno de Raynaud, distrofia cutánea hasta la evidencia de úlceras $(1,3)$ sin embargo no existe franca correlación entre los niveles séricos de crioglobulinas y la actividad de la enfermedad (3).

Otra manifestación clínica de la crioglobulinemia es la presencia de vasculitis crioglobulinémica que puede presentarse con síntomas generales como la debilidad, fiebre o artralgias, con evidencia de lesiones purpúricas y dolor articular, pudiendo cursar con factor reumatoide positivo pero con anticuerpos anticitrulínicos negativo, como se documentó en nuestro caso.

Se debe realizar enfásis en las manifestaciones cutáneas de la crioglobulinemia mixta, dado que son síntomas típicos descritos la presencia de púrpura ortostática intermitente hasta lesiones úlcerosas en piernas y especialmente en área maleolar con cambios de coloración ocre en dichas áreas $(5,6)$. Estos hallazgos son evidentes en las imágenes previamente expuestas en el artículo.

Posteriormente se debe revisar el compromiso renal surgido en la crioglobulinemia lo cual se ha descrito hasta en un 20\% de los pacientes, observando un espectro desde la presencia de proteinuria con síndrome nefrótico, hematuria con o sin síndrome nefrótico hasta la falla renal progresiva $(1,7,8)$.

Dentro de los hallazgos histológicos documentados en la biopsia renal de pacientes con crioglobulinemia sobresalen la presencia de glomerulonefritis membranoproliferativa, $y$ en otras oportunidades la presencia de glomeruloefritis 
mesangioproliferativa y focal, o glomerulonefritis membranosa (8). Incluso se han documentado reportes de casos de miocroangiopatía trombótica en algunos pacientes (9). Sin embargo el mecanismo fisiopatológico que establezca las causas del depósito de complejos de crioglobulinas dentro del riñón no se ha logrado establecer de forma clara, aunque pueden influir factores hemodinámicos, así como la habilidad de unir y activar componentes del complemento como el C1q (10).

Del mismo modo, también se ha descrito el compromiso pulmonar en los pacientes con esta entidad, observando la presencia de disnea y tos seca, sumado a cambios de fibrosis pulmonar, con evidencia en el lavado broncoalveolar de predominio de macrófagos y linfocitos, e inclusive la presencia de hemorragia alveolar documentada por la presencia de hemoptisis, falla respiratoria con infiltrados difusos pulmonares, documentando la presencia de hemosiderófagos en el lavado broncoalveolar $(1,11,12)$. Dicho compromiso por alveolitis se ha considerado secundario a la presencia de complejos inmunes (13).

Adicionalmente existen diferentes compromisos de la enfermedad en otros sístemas como el nervioso donde se puede documentar la presencia de neuropatía periférica descrita entre un 17 a $60 \%$ de los pacientes con crioglobulinemia, o la presencia de eventos isquémicos (1), o inclusive compromiso gastrointestinal por vasculitis que puede simular colecistitis. Igualmente, existe compromiso multisistémico asociado a la presencia de crioglobulinemia, como la presencia de síndrome pulmón - riñón, el cual se caracteriza por hemorragia alveolar difusa y glomerulonefritis con la presencia de falla renal (14).

\section{Conclusiones}

La crioglobulinemia hace parte de las entidades desencadentantes tanto del síndrome pulmón - riñón, así como de hemorragia alveolar, sin embargo se trata de un diagnóstico de exclusión. A través del presente artículo se ha revisado el caso de una paciente con úlcera y lesiones petequiales que cursaba con deterioro progresivo de la función renal y posterior hemorragia alveolar generando falla respiratoria, documentando la presencia de crioglobulinas y descartando etiología autoinmune, neoplásica e infecciosa.

\section{Conflictos de intereses}

Los autores declaran no tener de manera directa o indirecta, ningún tipo de conflicto de intereses financieros, académicos o laborales que puedan poner en peligro la validez de este estudio.

\section{Financiación}

El presente trabajo no tuvo financiación externa de ninguna entidad pública o privada.

\section{Referencias}

1. Ramos-Casals M, Stone JH, Cid MC, Bosch X. The cryoglobulinaemias Lancet. 2012 28;379(9813):348-360.

2. Cacoub P, Costedoat-Chalumeau N, Lidove O, Alric L. Cryoglobulinemia vasculitis. Curr Opin Rheumatol. 2002;14(1):29-35.

3. Tedeschi A, Baratè C, Minola E, Morra E. Cryoglobulinemia Blood Reviews. 2007; 21(1): 183-200.

4. Adinolfi LE, Utili R, Attanasio V, Zampino R, Ragone E, Tripod MF, Ruggiero G. Epidemiology, clinical spectrum and prognostic value of mixed cryoglobulinaemia in hepatitis $C$ virus patients: $a$ prospective study. Ital J Gastroenterol. 1996;28(1):1-9.

5. Mixed Cryoglobulinemia en: Handbook of systemic autoimmune diseases, Volume 5 The skin in systemic autoimmune diseases. Elsevier B.V. 2006.p: $272-274$.

6. Cakir O, Ayyildiz O, Isikdogan A. Type III mixed cryoglobulinemia associated with digital necrotic ulcer successfully treated with intermittent intravenous pulse cyclophosphamide. Angiology. 2005;56(4):489-492.

7. Beddhu S, Bastacky S, Johnson JP. The Clinical and Morphologic Spectrum of Renal Cryoglobulinemia. Medicine (Baltimore). 2002;81(5):398-409.

8. Mationon M, Cacoub P, Colombat M, Saadoun D, Brocheriou I, Mougenot B et al. Clinical and morphologic spectrum of renal involvement inpatients with mixed cryoglobulinemia without evidence ofhepatitis $\mathrm{C}$ virus infection. Medicine (Baltimore). 2009;88(6):341-348.

9. Herzenberg AM, Telford JJ, De Luca LG, Holden JK, Magil AB Thromobotic microangiopathy associated with cryoglobulinemic membranoproliferative glomerulonephritis and hepatitis C. Am J Kidney Dis. 1998;31(3):521-526.

10. Alpers CE, Smith KD. Cryoglobulinemia and renal disease. Curr Opin Nephrol Hypertens. 2008;17(3):243-249.

11. Perelló Carbonel R, Supervía Caparrós A, Nolla Salas J, Vázquez Sánchez A, Torrente Segarra V,Gutiérrez Cebollada J. Alveolar haemorrhage and hepatitis virus $\mathrm{C}$ related mixed cryoglobulinemia. Report of three cases. An Med Interna. 2005;22(11):529-31.

12. Bertorelli G, Pesci A, Manganelli P, Schettino G, Olivieri D. Subclinical pulmonary involvement in essential mixed cryoglobulinemia assessed by bronchoalveolar lavage. Chest. 1991;100(5):1478-1479.

13. Amital H, Rubinow A, Naparstek Y. Alveolar hemorrhage in cryoglobulinemia an indicator of prognosis.IClin Exp Rheumatol. 2005;23(5):616-620.

14. McCabe C, Jones Q, Nikolopoulou A, Wathen C, Luqmani R Pulmonary-renal syndromes: An update for respiratory physicians. Respir Med. 2011;105(10):1413-1421. 